\title{
Biodiversity and Plant Growth Promoting Potential of Bacteria from Soybean Rhizosphere of Saline Soil
}

\author{
Dhanraj Nakade \\ Government of Maharashtra, Elphinstone College, Dr. Homi Bhabha University, Mumbai, Maharashtra, India
}

\section{Email address:}

nakadedhanraj@gmail.com

\section{To cite this article:}

Dhanraj Nakade. Biodiversity and Plant Growth Promoting Potential of Bacteria from Soybean Rhizosphere of Saline Soil. American Journal of Agriculture and Forestry. Vol. 8, No. 3, 2020, pp. 77-80. doi: 10.11648/j.ajaf.20200803.14

Received: April 5, 2020; Accepted: April 24, 2020; Published: June 12, 2020

\begin{abstract}
PGPR are root-associated bacteria that form symbiotic relationships with many plants. These are the Bacteria that colonize plant roots and promote plant growth are referred to as plant growth-promoting rhizobacteria (PGPR). PGPR are highly diverse and are important in plant growth promotion and increase in yield of crops. Almost all of the PGPR bacteria produce phytohormones, some fixes atmospheric nitrogen, some solublizes the phosphates and some resist phytopathogens by production of siderophores. An understanding of microbial diversity is important in agricultural contest, it is important and useful to know soil quality in terms of PGPR bacteria which is helpful for taking measures for soil management and increased plant productivity. It is also important to understand the relationship of soil and plants with the diversity of associated bacteria for their better exploitation. Therefore, it is important to know the soil micro flora and their diversity. Most of the rhizospheric bacterial diversity from normal soil have been studied and organisms explored for their use as bioinoculents. However, saline soil rhizospheric microfloras remain unexplored. By considering this, in the present study fifty two bacterial isolates including PGPR have been isolated from saline soil of Kolhapur district of southern Maharashtra, India. Isolates were identified up to genus and species level. Few isolates were studied their nitrogen fixing and phosphate solublizing activity. Present study showed that amongst nitrogen fixing bacteria Azotobacter chroococcum found to be most dominant and Bacillus megaterium was found to be most dominant phosphate solublizer. Study indicated the importance of these organism as bioinoculents for saline soil and can be explored for biofertilizer production.
\end{abstract}

Keywords: Diversity, PGPR, Saline Soils, Rhizosphere, Soy Bean

\section{Introduction}

Salinity "A major stress limiting agriculture productivity"

On the global basis salt affected soils occupy an estimated 952.2 million hectares of land, constituting $7 \%$ of total land affected by salinity [1] The problem of soil salinity is wide spread in the world, amongst the affected country, Holland, Swedan, Hungary, Russia, South western USA, India, Pakistan and the Middle east are worstly affected. About 40,000 hectares of land annually becoming unfit for agricultural production in the world due to salinity.

In India the the problem has taken a serious mode about $9 \%$ of the total cultivated area is affected by salinity [2]. The problem is acute in the state of Maharashtra, Punjab, Hariyana and Uttar Pradesh states of India.

In Maharashtra about 34 million hectare has become salt affected. Such soils are predominant in Kolhapur, Sangli,
Solapur, Ahmednagar, Dhule districts of Maharashtra state of India.

\subsection{Approaches to Combat Salinity}

1) Chemical amendment

2) Development of salt tolerant plants through breeding/genetic engineering.

3) Use of PGPR Microorganisms- A viable approach; use of salt tolerant microbes to induce tolerance in plants, economical, sustainable \& environment friendly.

\subsection{Tolerance Limit}

1) Threshold level of salt tolerance in plants varies from 40-200mM NaCl. 
2) Tolerance level of PGPR varies from 100-650mM NaCl.

\subsection{Role of Pgpr}

a) Better development of root system

b) Production of growth promoting hormones in addition to stress hormone ABA.

c) Solubilization of insoluble phosphate

By considering this in the present study total of 52 Plant Growth Promoting rhizobacteria was isolated from Soybean rhizosphere of saline soil. Isolates were isolated using different media and screened for plant growth promoting (PGP) activities at higher salt $(\mathrm{NaCl})$ concentrations $2 \%, 4 \%, 6 \%, 8 \%, 10 \%$.

\section{Material and Methods}

\subsection{Collection of Samples [3]}

Soil adhered to roots of Soybean plant from saline soils were collected from fourty different sites in sterile plastic bags from Kolhapur district of Maharashtra, India.

One gram rhizospheric soil sample was dissolved in $100 \mathrm{ml}$ of buffered saline and placed on shaker for $30 \mathrm{~min}$, From this different dilutions viz 10-2, 10-4, 10-6, 10-8, 10-10 were prepared. From each dilutions $0.1 \mathrm{ml}$ was spread Nutrient agar for isolation as well as enumeration of different bacteria, $0.1 \mathrm{ml}$ was spread on AshbysMannitol agar for Azotobacter spp., Congored yeast extract agar for Rhizobium spp., Nitrogen free agar for Azospirillumspp respectively. Individual colonies showing different morphology from respective medium were transferred on slants of respective media and further used for identification and other studies. Unless otherwise stated experiment was conducted in triplicates.

All the isolates were identified as per [4] Vol. I, II, III, IV, $\mathrm{V}, \mathrm{VI}$ and [5].

\subsection{Screening of Plant Growth Promoting Bacteria}

\subsubsection{Phosphate - Solublization}

Phosphate- solublization was detected qualitatively by spot inoculation of isolates [6], containing Glucose $10 \mathrm{~g}$, Tribasic phosphate $5 \mathrm{~g},\left(\mathrm{NH}_{4}\right)_{2} \mathrm{SO}_{4}-0.5 \mathrm{~g}, \mathrm{KCl}-0.2 \mathrm{~g}, \mathrm{MgSO}_{4} \cdot 7 \mathrm{H}_{2} \mathrm{O}-0.1 \mathrm{~g}$, trace of $\mathrm{MnSO}_{4}$ and $\mathrm{FeSO}_{4}$, Yeast extract $0.5 \mathrm{~g}, \mathrm{NaCl} 4 \%$, Agar Agar 15 g, Distilled water 1000 ml, pH-7.0. After incubation at room temperature for 48 hours a clear zone around colony was used as indicator for positive phosphate

\subsubsection{Nitrogen Fixation}

Nitrogen fixation was detected by Acetylene reduction assay [7], using a chemically defined medium containing $\mathrm{K}_{2} \mathrm{HPO}_{4} 0.60 \mathrm{~g}^{-1}, \mathrm{KH}_{2} \mathrm{PO}_{4} 0.14 \mathrm{~g}^{-1}$,

$\mathrm{MgSO}_{4} .7 \mathrm{H}_{2} \mathrm{O} 0.2 \mathrm{~g}^{-1}, \mathrm{FeSO}_{4} .7 \mathrm{H}_{2} \mathrm{O} 0.44 \mathrm{~g}^{-1}, \mathrm{ZnSO}_{4} .7 \mathrm{H}_{2} \mathrm{O}$ $0.00028 \mathrm{~g}^{-1}, \mathrm{H}_{2} \mathrm{BO}_{3} 0.0032 \mathrm{~g}^{-1}, \mathrm{Na}_{2} \mathrm{MoO}_{4} .2 \mathrm{H}_{2} \mathrm{O} 0.003 \mathrm{~g}^{-1}$, $\mathrm{MnSO}_{4} \cdot \mathrm{H}_{2} \mathrm{O} 0.004 \mathrm{~g}^{-1}, \mathrm{NaCl} 4 \%$, Sucrose $20 \mathrm{~g}^{-1}$

\subsubsection{Indole Acetic Acid Production}

Indole acetic acid produced by isolates was assayed colorimetrically using Ferric chloride-perchloric acid reagent [8]

For this isolates were grown in $50 \mathrm{ml}$ modified nutrient broth inoculated with $4 \% \mathrm{NaCl}$ salt for 24 hours on rotary shaker at $150 \mathrm{rpm}$ and room temperature and used as seed culture. From this $100 \mathrm{ul}$ of was inoculated in $10 \mathrm{ml}$ minimal salt (MS) medium containing $\mathrm{KH}_{2} \mathrm{PO}_{4}-0.136, \mathrm{Na}_{2} \mathrm{HPO}_{4}-$ $0.213 \mathrm{~g}, \mathrm{MgSO}_{4} .7 \mathrm{H}_{2} \mathrm{O}-0.02 \mathrm{~g}$, Trace element solution 0.001 , Tryptophan $0.5 \mathrm{mM}$, NaCl-4 g, Distilled water-100 ml, $\mathrm{pH}-$ 7.0., [9].

After incubation at room temperature for 48 hours, $1.5 \mathrm{ml}$ broth culture was centrifuged at $12000 \mathrm{rpm}$ for 5 minutes. One $\mathrm{ml}$ supernatant was added to $2 \mathrm{ml} \mathrm{FeCl} 3-\mathrm{HClO}_{4}$ reagent. After 25 minutes (once color density reaches maximum) the mixture was read in UV-spectrophotometer at $530 \mathrm{~nm}$ absorbance. The amount of IAA produced per ml culture was estimated using a standard curve.

\subsubsection{Siderophore Production}

It was assayed [10] Isolates producing an orange halo zone around growth on Chromeazurol S agar (CAS) after 48-72 hours of incubation were considered as positive.

\section{Result and Discussion}

Table 1. Indicates the list of identified bacteria from Soybean rhizosphere of saline soils. Amongst all the bacterial isolates genera Bacillus was found to be the most dominant followed byPseudomonas which correlates with Gaur et al., [11] List of Identified Bacterial isolates.

\begin{tabular}{|c|c|c|c|c|c|}
\hline Isolate No. & Name of the bacterial Isolate & Isolate No.nNo. & Name of the bacterial Isolate & Isolate No. & Name of the bacterial Isolate \\
\hline 1 & Bacillus subtilis & 21 & Pseudomonas pinophilum & 41 & Pseudomonas alcaligens \\
\hline 2 & Bacillus brevis & 22 & Pseudomonas putida & 42 & Pseudomonas pseudoalcaligens \\
\hline 3 & Bacillus cereus & 23 & Pseudomonas stutzeri & 43 & Bacillus pumilis \\
\hline 4 & Bacillus circulans & 24 & Serratiaphosphaticum & 44 & Bacillus pulvifaciens \\
\hline 5 & Rhizobiumspecies & 25 & Azotobactorchroococcum & 45 & Azoarcuscommunis \\
\hline 6 & Azospirillumlipoferum & 26 & Serratiamarcescens & 46 & Flavobacterium species \\
\hline 8 & Methylobacterium species & 28 & Escherichia freundii & 48 & Paenibacilluspolymyxa \\
\hline 9 & Pseudomonas fluorescens & 29 & Bacillus mesentricuc & 49 & Alcaligenesxylosoxidans \\
\hline 10 & Pseudomonas pseudomallei & 30 & Bacillus mycoides & 50 & Pseudomonas striata \\
\hline 11 & Alcaligenes species & 31 & Bacillus pumilis & 51 & Micrococcus luteus \\
\hline 12 & Arthrobacter species & 32 & Azomonas species & 52 & Serratamarcescens \\
\hline 13 & Azotobactorvenelandii & 33 & Corynebacterium species & & \\
\hline
\end{tabular}




\begin{tabular}{|c|c|c|c|c|c|}
\hline Isolate No. & Name of the bacterial Isolate & Isolate No.nNo. & Name of the bacterial Isolate & Isolate No. & Name of the bacterial Isolate \\
\hline 14 & Azospirillumbrasilens & 34 & Rhodospirillum species & & \\
\hline 15 & Azospirillumhalopraeferens & 35 & Rhodopseudomonas species & & \\
\hline 16 & Bacillus circulans & 36 & Azotobacterbeijerinkii & & \\
\hline 17 & Bacillus megaterium & 37 & Azotobacternigricans & & \\
\hline 18 & Bacillus firmus & 38 & Aeromonas species & & \\
\hline 19 & Bacillus licheniformis & 39 & Acetobacter species & & \\
\hline 20 & Pseudomonas cissicola & 40 & Pseudoxanthomonas species & & \\
\hline
\end{tabular}

Table 2. Isolates producing (IAA), P-solublization, Nitrogen fixation, and Siderophore production.

\begin{tabular}{|c|c|c|c|c|c|c|c|c|c|c|c|c|c|c|}
\hline Strain no. & (A) & (B) & (C) & (D) & Strain no. & (A) & (B) & (C) & (D) & Strain no. & (A) & (B) & (C) & (D) \\
\hline $\mathrm{N}-1$ & - & + & - & - & $\mathrm{N}-21$ & 6.2 & - & - & - & $\mathrm{N}-41$ & 7.2 & - & - & - \\
\hline $\mathrm{N}-2$ & - & + & - & - & $\mathrm{N}-22$ & - & - & - & + & $\mathrm{N}-42$ & - & + & - & - \\
\hline $\mathrm{N}-3$ & - & + & - & - & $\mathrm{N}-23$ & - & - & - & + & $\mathrm{N}-43$ & 15.3 & - & - & - \\
\hline $\mathrm{N}-4$ & - & + & - & - & $\mathrm{N}-24$ & 20.4 & - & - & - & $\mathrm{N}-44$ & - & + & - & - \\
\hline N-5 & - & - & + & - & $\mathrm{N}-25$ & - & - & + & - & $\mathrm{N}-45$ & - & + & - & - \\
\hline N-6 & - & - & + & - & $\mathrm{N}-26$ & 12.3 & - & - & - & $\mathrm{N}-46$ & - & + & - & - \\
\hline $\mathrm{N}-7$ & - & - & + & - & $\mathrm{N}-27$ & - & - & - & + & N-47 & - & - & - & + \\
\hline $\mathrm{N}-8$ & 24.5 & - & - & - & $\mathrm{N}-28$ & - & + & - & - & N-48 & - & - & - & + \\
\hline N-9 & 6.3 & - & - & - & $\mathrm{N}-29$ & - & + & - & - & $\mathrm{N}-49$ & 31.2 & - & - & - \\
\hline $\mathrm{N}-10$ & 28.2 & - & - & - & $\mathrm{N}-30$ & - & + & - & - & N-50 & - & - & - & - \\
\hline $\mathrm{N}-11$ & - & - & - & + & $\mathrm{N}-31$ & - & + & - & - & N-51 & - & - & - & - \\
\hline $\mathrm{N}-12$ & 17.9 & - & - & - & $\mathrm{N}-32$ & - & + & - & - & $\mathrm{N}-52$ & - & - & - & - \\
\hline $\mathrm{N}-13$ & - & - & + & - & $\mathrm{N}-33$ & 9.4 & - & - & - & & & & & \\
\hline $\mathrm{N}-14$ & - & - & + & - & N-34 & - & - & - & - & & & & & \\
\hline $\mathrm{N}-15$ & - & - & + & - & $\mathrm{N}-35$ & - & - & - & - & & & & & \\
\hline $\mathrm{N}-16$ & 4.7 & - & - & - & $\mathrm{N}-36$ & - & - & + & - & & & & & \\
\hline $\mathrm{N}-17$ & - & + & - & - & N-37 & - & - & + & - & & & & & \\
\hline $\mathrm{N}-18$ & - & + & - & - & $\mathrm{N}-38$ & - & - & - & - & & & & & \\
\hline N-19 & - & + & - & - & $\mathrm{N}-39$ & - & - & - & - & & & & & \\
\hline $\mathrm{N}-20$ & - & + & - & - & N-40 & - & - & - & - & & & & & \\
\hline
\end{tabular}

(A) IAA production $\left(\mu \mathrm{mol} \mathrm{ml}^{-1}\right.$, (B) P-solublization, (C) $\mathrm{N}_{2}$-fixation, (D) Siderophore production, $(+)$ positive, (-) negative

The strains from the genera Bacillus, Pseudomonas, Rhizobium are amongst the most phosphate solublizers. Genera Pseudomonas was dominant [12-16]. Rodriguez and Fraga, studied the Soybean PGPR and their role in plant growth promotion. They found that Azotobacter chroococcum as most dominant Nitrogen fixer and Bacillus megaterium as most dominant phosphate solublizer [17]. I report Pseudomonas fluorescens as most dominant phosphate solublizer and Azotobacter chroococcum as dominant Nitrogen fixer.

Of all the 52 isolates 12 produced Indole acetic acid (IAA), 17 solublized phosphates, 9 fixed Nitrogen, 6 produced siderophores,

The overall results showed that only 8 isolates did not show any of the four PGPR traits. The amount of IAA produced by some isolates N49 was higher (31.2) than that have been reported $[18,19,20]$, which range from 2.31 to $9.43 \mu \mathrm{mol} \mathrm{ml}{ }^{-1}$. Further study is required to utilize potential application for high IAA production.

\section{Conclusion}

All the isolates tolerated $8 \% \mathrm{NaCl}$ concentration, grows optimally at $4 \% \mathrm{NaCl}$, hence they have a potential to be used as bioinoculents for saline soils.

\section{Acknowledgements}

I am very much thankful to our Principal, Elphinstone college, Mumbai and Director Institute of Science, Aurangabad for making me the laboratory available to carry out my research.

\section{References}

[1] Bresler E., McNeal B. L., Carter D. L., (1982) Saline and sodic soils, Principles-dynamics-modelling' (Springer-Verla, Berlin, HeidelbergEds), New York, pp. 206-223.

[2] Dahiya IS., Dahiya SS., (1977) Salt affected soils in India: I Their origin, occurrence and characteristics', J. Aric. Agro. Indus, 10 (1) pp. 11-19.

[3] Richards L. A., Diagnosis and improvement of Saline alkali soil, USDA Handbook No. 60. Wasington, 69-82 (1954).

[4] Williams ST; Sharpe ME; Holt TJ, Bergey's manual of systematic bacteriology, Vol. I, II, III, IV, The Williams and Wilkins co. Baltimore. 1989.

[5] Portyrata D A; Krichevosky MI, MICRO-IS, a microbiological database management and analysis system, Binary, 1992, 4: 31-36. 
[6] Subba Rao N. s., (1999) Soil Microbiology (Fourth edition of Soil Microorganisms and plant growth) Science Publishers, Inc. USA.

[7] Dobereiner J, Soil boilBiochem, 1997, 29: 771-774.

[8] Hardy RR; Burns WF; Holston RD, Soil. Biol. Biochem, 1975, 2, 47-81.

[9] Frankenberger W. T., Poth M., (1988) L-tryptophan transaminase of a bacterium isolated from the rhizosphereof Festucaoctofora (Gramineae) Soil BiolBiochem, 20: 299-304.

[10] Gorden S. A., Weber R. P., (1951) Colorimetric estimation of indole acetic acid, Plant physiol, 26: 192-197.

[11] Lee K. D., Bai Y., Smith D., Han H. S., Supanjani, (2005) Isolation of Plant growth promoting endophytic bacteria from bean nodule, Res. J. Agric. Biol. Sci., 1 (3): 232-236.

[12] Schwyne B., Neialnds J. B., (1987) Annual. Biochem. 160: 40-47.

[13] Gaur R., Shani N., Kawaljeet-Johri B. N., Rossi P., Aragno M., (2004) Curr. Sci., 86: 453-457.

[14] Koide R. T., (1991) Nutrient supply, nutrient demand and plant response to Mycorrhizal infection, Newphytol, 117: 365386.
[15] Jetiyanon K., Fowler W. D., Kloepper J. W., (2003) Broadspectrumprotection against several pathogens by PGPR mixtures under field conditions., Plant Dis, 87: 1390-1394.

[16] Vessey J. K., (2003) Plant growth promoting rhizobacteriaas biofertilizers, Plant-Soil, 255: 571-586.

[17] Bashan Y., Holguin G., De-Bashan L. E. (2004) AzospirillumPlant relationships, physiological, Molecular, Agricultural and Environmental advances (1997-2003)., Can J. Microbiol., 50: 521-577.

[18] Wu S. C., Cao Z. H., Li Z. G., Cheung K. C., Wong M. H., (2005) Effects of biofertilizer containing N-fixer, P and K solublizers and AM fungi on maize growth: a green house trial, Goderma, 125: 155-166.

[19] Rodriguez H., Fraga R., (1999) Phosphate solublizing bacteria and their role in plant growth promotion., Biotechnol Adv, 17: 319-339.

[20] De Freitas J. R., Banerjee M. R., Germida J. J., (1997) Phosphate-solublizingrhizobacteria enhance the growth and yield but not phosphorous uptake of Canola (Brassica napus L.) Biol. Fertil. Soils, 24: 358-364. 